\title{
DIII-D THOMSON SCATTERING DIAGNOSTIC DATA ACQUISITION, PROCESSING, AND ANALYSIS SOFTWARE
}

\author{
by \\ K.R. MIDDAUGH, B.D. BRAY, C.L. HSIEH, B.B. McHARG, Jr., \\ and B.G. PENAFLOR
}

This is a preprint of a paper to be presented at the 11th IEEE/NPSS Real Time Conference, June 14-18, 1999, Santa Fe, New Mexico and to be published in Transactions on Nuclear Science.

\author{
Work supported by \\ the U.S. Department of Energy \\ under Contract No. DE-AC03-99ER54463
}

GA PROJECT 30033

JUNE 1999 


\section{DISCLAIMER}

This report was prepared as an account of work sponsored by an agency of the United States Government. Neither the United States Government nor any agency thereof, nor any of their employees, make any warranty, express or implied, or assumes any legal liability or responsibility for the accuracy, completeness, or usefulness of any information, apparatus, product, or process disclosed, or represents that its use would not infringe privately owned rights. Reference herein to any specific commercial product, process, or service by trade name, trademark, manufacturer, or otherwise does not necessarily constitute or imply its endorsement, recommendation, or favoring by the United States Government or any agency thereof. The views and opinions of authors expressed herein do not necessarily state or reflect those of the United States Government or any agency thereof. 


\section{DISCLAIMER}

Portions of this document may be illegible in electronic image products. Images are produced from the best available original document. 


\title{
DIII-D Thomson Scattering Diagnostic Data Acquisition, Processing, and Analysis Software*
}

\author{
K.R. Middaugh, B.D. Bray, C.L. Hsieh, B.B. McHarg, Jr., B.G. Penaflor \\ General Atomics, P.O. Box 85608, San Diego, California 92186-5608
}

\section{Abstract}

One of the diagnostic systems critical to the success of the DIII-D tokamak experiment is the Thomson scattering diagnostic. This diagnostic is unique in that it measures local electron temperature and density: 1) at multiple locations within the tokamak plasma; and 2) at different times throughout the plasma duration.

Thomson "raw" data are digitized signals of scattered light, measured at different times and locations, from the laser beam paths fired into the plasma. Real-time acquisition of this data is performed by specialized hardware. Once obtained, the raw data are processed into meaningful temperature and density values which can be analyzed for measurement quality.

This paper will provide an overview of the entire Thomson scattering diagnostic software and will focus on the data acquisition, processing, and analysis software implementation.

The software falls into three general categories:

1. Set-up and Control: Initializes and controls all Thomson hardware and software, sychronizes with other DIII-D computers, and invokes other Thomson software as appropriate.

2. Data Acquisition and Processing: Obtains raw measured data from memory and processes it into temperature and density values.

3. Analysis: Provides a graphical user interface in which to perform analysis and sophisticated plotting of analysis parameters.

\section{INTRODUCTION}

The DIII-D tokamak is a magnetic fusion research experiment operated by General Atomics (GA) under contract with the Department of Energy. This experiment is dedicated to the study of the properties of high temperature plasmas approaching fusion reactor-like plasma conditions. Results of these experiments are particularly applicable to next generation fusion devices [1]. During experiments, high temperature plasmas are created in the tokamak with a typical duration of $5 \mathrm{~s}$. Numerous diagnostic systems are used to monitor a variety of plasma parameters.

As one of the most critical diagnostic systems at DIII-D, the Thomson Scattering diagnostic is used to measure the temperature and density of the plasma [2]. The DIII-D Thomson Scattering system is unique in that it is capable of taking measurements at multiple locations within the plasma throughout the entire plasma duration. Currently there are forty-four spatial points being measured and eight lasers firing up to a total of 160 times per second. The "raw" measured data are actually digitized signals of scattered light that are taken at different spatial locations on laser beam paths fired into the plasma. The raw measurements are acquired by specialized real time hardware. The raw data is then processed by several software programs into meaningful temperature and density values which can be analyzed and studied just moments after the actual plasma discharge.

In this paper, an overview of the Thomson scattering diagnostic system will be presented which will focus on the software used for data acquisition, processing, and analysis.

\section{HARDWARE OVERVIEW}

The following is a brief overview of the hardware described in Fig. 1.

When a pulse of laser light is fired through a plasma, photons are scattered by the energetic plasma electrons. The scattered light is frequency shifted. The amount of frequency shift is temperature dependent and the total amount of light scattered is proportional to the electron density [2]. Thus light

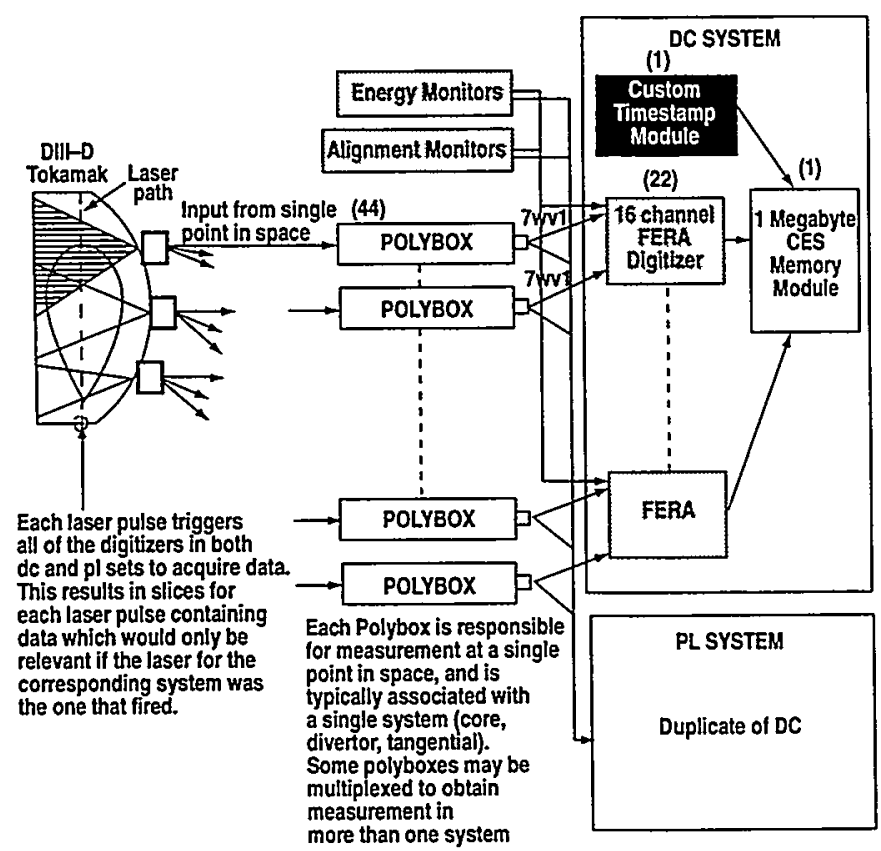

Fig. 1. Thomson data acquisition hardware overview.

\footnotetext{
${ }^{*}$ Work supported by U.S. Department of Energy under Contract No. DE-AC03-99ER54463.
} 
frequency shift and total scattered light measurements can be used to calculate the plasma temperature and density.

Within the DIII-D vacuum vessel, there are three laser paths used to make measurements in three regions (Fig. 2). One vertical path is used for divertor, or lower region measurements. Another vertical path is used for main core or main plasma measurements, excluding the central region. The horizontal path is used for the central core measurements. The horizontal path was recently added in order to provide measurements closer to the center of the plasma.There are eight Nd:YAG lasers distributed among the three paths.

Forty-four optical fibers are "aimed" at the laser paths and each is plugged into one of the forty-four polychromator detectors [3]. Light is detected by silicon avalanche photo diode detectors [4]. Gain values of detectors are specified via six VMIC 128-bit TTL input/output VME boards [5]. The detector's output signals are digitized by FERA CAMAC modules [6]. Two Creative Electronic Systems (CES) 1 MByte VME memory boards [7] are used to store the measurements.

\section{SOFTWARE: OVERVIEW AND SET UP AND CONTROL}

During plasma experiments, the Thomson control program coordinates the Thomson diagnostic with the rest of the DIII-D experiment, initializes the hardware prior to the plasma shot, and controls the after shot software processing.

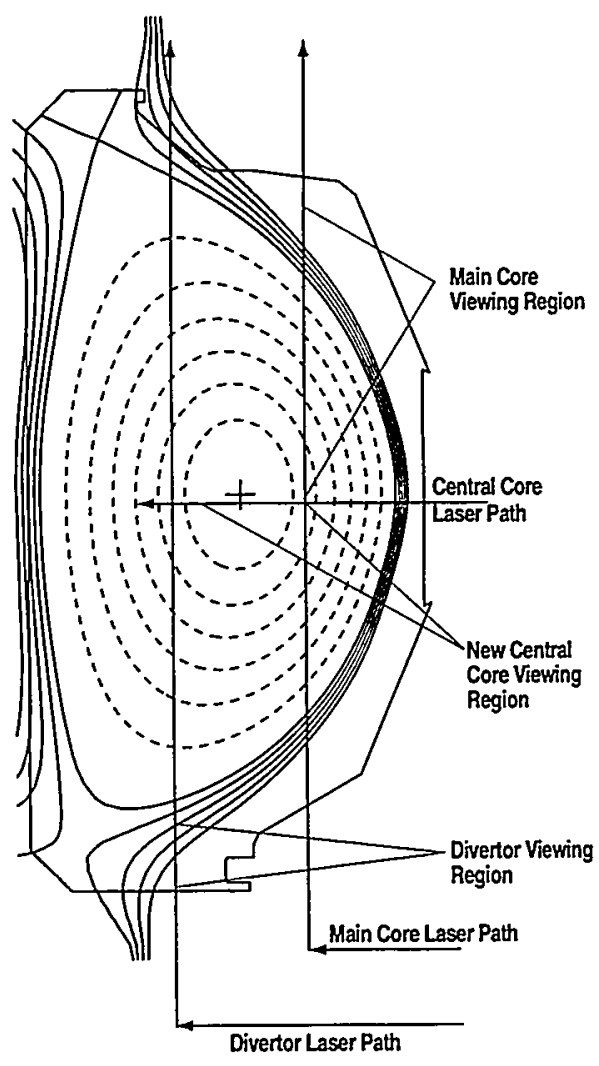

Fig. 2. DIII-D Thomson laser paths.
This program is implemented with Vista Control Systems, Inc. Vsystem and Vaccess [8] software and currently runs on a DEC Alpha under Open VMS. The Vdraw [8] utility allowed the creation of an attractive Graphical User Interface (GUI). Vaccess is a real time database used to represent various Thomson states and values. The Thomson control program continually monitors the current state of the system. As "triggers" arrive from the DIII-D outside world before and after a plasma shot, the GUI will update its display to indicate appropriate status to the operator, will initialize the Thomson hardware for light measurement and will activate numerous other programs to perform desired tasks. Once the light measurements reside in memory after the plasma shot has completed, the control program initiates the data acquisition software, and the temperature and density profile generation software.

Figure 3 shows an overview of the progression of data through the Thomson system. The Thomson Scattering Data Acquisition (TSDACQ) program will obtain the digitized light signals from memory and sort the data into appropriate core, tangential, and divertor systems. The resulting data is referred to as sorted data. The Thomson Scattering (temperature and density) Profile (TSPROF) generator takes the sorted data as input along with calibration data, and calculates temperature and density profiles within the core, tangential, and divertor systems. The Thomson Scattering Data Analysis (TSDAT) GUI can then be used to analyze the temperature and density profiles in order to determine the quality of the resultant measurements.

\section{SOFTWARE: DATA ACQUISITION - TSDACQ}

TSDACQ is a program and associated library written in the $\mathrm{C}$ language. It acquires the raw light signal data and processes it into data sorted by the core, tangential, and divertor systems by the corresponding spatial positions. This sorting requires several steps.

Before the polychromator detectors can measure background light, they must have their background measurement circuits set to one of the possible four gain values. Therefore before every plasma shot, the control program initiates TSDACQ to set these gain values. The current gain values are stored in an ASCI file and are read by TSDACQ. Then TSDACQ sets the outputs of the VMIC TTL board which in turn set the gain values of the background measurement circuits since the outputs of the TTL board are wired to the circuits.

The control program then invokes TSDACQ to initialize the CES memory boards. Next, the control program initiates 200 measurements in the vacant tokamak without laser pulses to obtain dark current or "pedestal" measurements. The control program then initiates TSDACQ to acquire the dark current from the CES memory boards and store the raw data into two files: 1) pedestal-dc and 2) pedestal-pulse. Again the control program invokes TSDACQ to initialize the CES memory boards. 


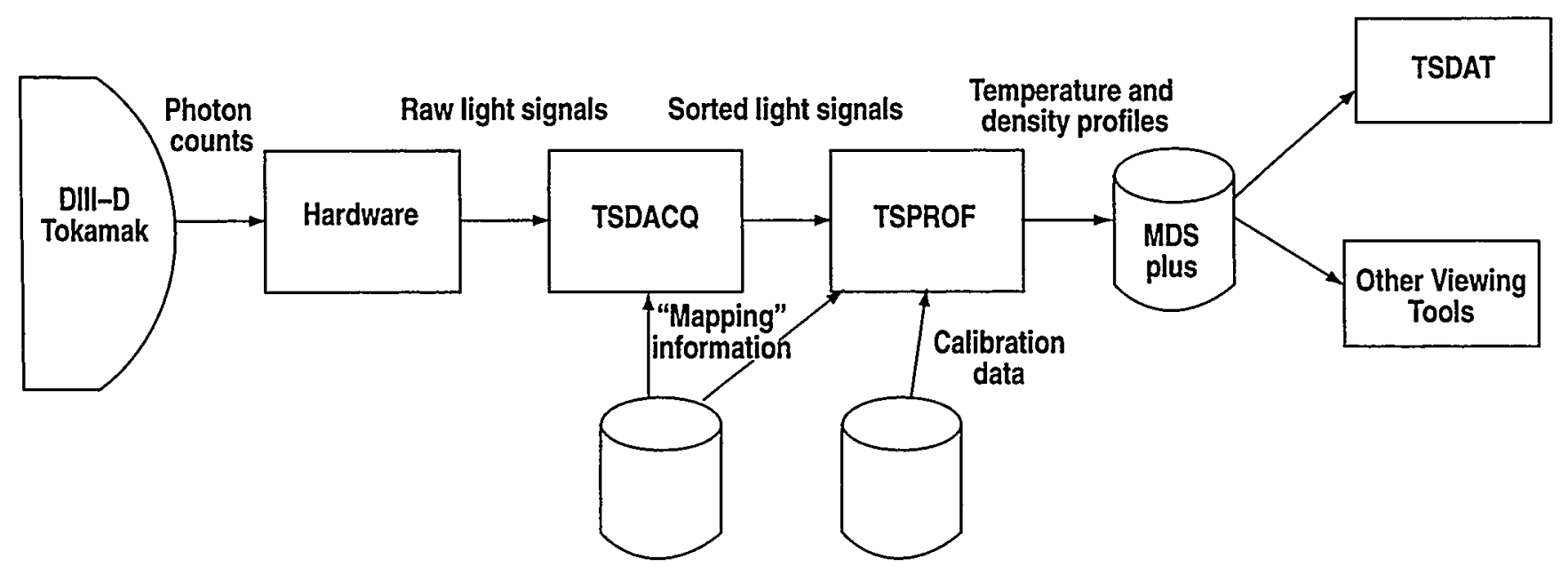

Fig. 3. Thomson data flow overview.

Then the plasma shot commences and the raw light signal or "shot" data gets acquired by the hardware and stored into memory. The control program again initiates TSDACQ to acquire the light signals from the CES memory boards and store the raw data into another two files. Each file divides or sorts the data into two categories. The "pulse" category is for light signals taken at the time a laser is firing. A "DC" category is for light signals taken before the laser is firing. Thus, these two raw light signal files are: 1) shot-dc and 2) shot-pulse.

Up to this point, the raw light signal data has been obtained from the CES memory and stored into four raw data files. The next step is to sort the raw data into the correct spatial position order for the core, tangential and divertor systems.

The control program invokes TSDACQ for the final time. The four raw data files and mapping information describing the spatial location and wavelength range of each digitizer channel are read into local memory. Using the mapping information, each light signal is ordered by system, spatial position, and time. This data set is known as the sorted data. Calibration data, gain values, and other miscellaneous values are assembled into a "header" data set. The header, raw, and sorted data sets are known as HRS data. Finally, TSDACQ writes the HR data to the DIII-D point database (PTDATA) for permanent storage, and writes the HRS data to a disk file for fast access by the TSPROF program.

\section{SOFTWARE: DATA PROCESSING - TSPROF}

TSPROF is a program and associated library also written in the $C$ language. Using the sorted data, hardware mapping data, and calibration data as inputs, TSPROF can calculate the temperature and density profiles.

After the raw light signal data has been successfully sorted, the control program will invoke the TSPROF program. The first task is to obtain all of the required input data. TSPROF will obtain the HRS data via a TSDACQ library routine. This HRS read routine first looks on the local disk for the HRS file. If it doesn't exist, it reads the HR data from PTDATA, then creates the S (sorted) data. Next, TSPROF will read the hardware mapping information and calibration information.

Once the required input data has been obtained, processing can begin. Since the Thomson diagnostic will take measurements before the plasma is created and continue until after the plasma has terminated, it is necessary to identify measurements that occurred before and after the plasma discharge. To perform this task, the plasma current measurements are read from the PTDATA database. Then for each measurement, the corresponding plasma current value is checked. If the plasma current value is less than a specified minimum, the measurement is marked to be ignored or "suppressed" from further calculations.

TSPROF determines the temperature and density by using a lookup table (LUT). There is one LUT for each polychromator that is created by using some of the calibration files. Each LUT contains expected digitized light signals for the temperatures of interest. Thus, the fit algorithm searches the LUT comparing a set of measured digitized light signals for the given polychromator with the expected values calculated in the LUT. It finds the best match or fit by employing a recursive minimization algorithm. Finally, the results are stored in the DIII-D MDSplus database system [9].

\section{SOFTWARE: DATA VIEWING - TSDAT}

The functionality described in this section is not yet fully implemented. Version 1 of TSDAT contains some of the described functionality, however this version operates too slowly and it is not used except as a tool to help define the next generation. Version 2 development is currently underway and is expected to have useful functionality by mid-Summer, 1999.

During experiments, plasma shots are taken every 15-20 minutes and physicists use the Thomson temperature and density profiles to plan the next shot. There are several tools available to them for viewing profile data stored in MDSplus 
as soon as it is created by TSPROF. The TSDAT graphical user interface utility however goes beyond simple profile viewing. It is used by the Thomson physicists for monitoring, analyzing, and troubleshooting the diagnostic. TSDAT is capable of displaying temperature and density profiles as well as most every facet of the data used to calculate the profiles. Other data includes subsets or slices of the profile data, raw data, calibration data, intermediate data, error values, parameters used for the TSPROF fitting, and statistics. TSDAT can initiate the recalculation of profiles utilizing modified inputs, and can perform statistical analysis on multiple shots.

Many utilities at DIII-D are implemented using IDL [10]. IDL has sophisticated plotting capabilities which are desirable for viewing large data sets which are stored as one, two, or three dimensional arrays. TSDAT is implemented using IDL and utilizes the TSDACQ and TSPROF C libraries using the IDL "call external" routine.

For any given plasma shot, TSDAT can read all data including calibration data from PTDATA or MDSplus databases, and from disk files. TSDAT allows multiple shots or multiple versions of the same shot to be simultaneously resident in memory and the operator may switch the display amongst the memory-resident shots as desired. The ability to maintain data for multiple shots is necessary for the reanalysis capability and for multiple shot statistical analysis.

The ability to reanalyze the profile data is extremely useful for determining and correcting problems within the diagnostic. Any specified version of a plasma shot may be read from MDSplus and stored into memory. Fitting parameters may be changed, then TSPROF can be invoked (reanalysis) to get a revised set of results. Each reanalysis generates another version of the plasma shot data in memory. If desired, any version of results in memory may be saved to MDSplus for access by all physicists. Also, any memory version may be deleted to make room for other versions. If a set of TSPROF input parameters is discovered to result in better profiles, the set may be applied to a range of shots for reanalysis with new permanent versions created and stored.

The ability to compare multiple shots will aid in the discovery of systematic errors. Statistical analysis can compare results of one spatial channel, one polychromator, or one polychromator's detector to help determine if results values are consistently too high or low.

\section{RECENT ACCOMPLISHMENTS AND FUTURE DIRECTIONS}

New versions of TSDACQ and TSPROF were introduced this year at the beginning of the 1999 plasma experiments campaign. The impetus for the major rewrite of these codes was to provide a simpler implementation. A simple code foundation was necessary because of the new functionalities desired. Both programs required the addition of the new tangential system which was also introduced at the start of 1999 experiments.

The new version of TSDACQ saw the elimination of shared memory. The old shared memory implementation was causing periodic problems so its elimination has resulted in a more robust program. The new version is substantially simpler, easier to understand, and more maintainable.

The new version of TSPROF was a port from FORTRAN to $C$. This was beneficial because of more resident $C$ experience. Major enhancements required for the TSDATTSPROF interface have and will be more easily accomplished in the $\mathrm{C}$ language. Speed improvements are future goals by organizing the software structure more efficiently, eliminating redundant data reads, and eliminating redundant loops.

Continued development of TSDAT is expected for at least another year. Version 2 development has just begun and many functionalities are already planned. As the utility gets used, there will undoubtedly be new functional requirements.

Another future goal is to eliminate any data storage on local disks and to migrate data to either the PTDATA or MDSplus databases. These databases provide robust, permanent, and archived data storage.

\section{REFERENCES}

[1] General Atomics Fusion Group, http://www.fusion. gat.com

[2] J.C. DeBoo, "Thomson Scattering at GA," http://fusion. gat.com/DNT/DNT14.HTM.

[3] T.N. Carlstrom, et al., GA web page "http:// fusion.gat.com/Products/diagnostics/Polychromator."

[4] T.N. Carlstrom, et al., Rev. Sci. Instrum. 63, 4902, (1992).

[5] VME Microsystems International Corporation (VMIC), Huntsville, Alabama, Model VMTVME-2528.

[6] LeCroy Corporation, Chestnut Ridge, NM, Models 4300B and 4301.

[7] Creative Electronic Systems S.A., Geneve, Switzerland, Model HSM 8170.

[8] Vista Control Systems, Inc., Los Alamos, NM, Products Vsystem, Vaccess, and Vdraw.

[9] J. Stillerman, T.W. Fredian, "The MDSplus Data Acquisition System Current Status and Future Directions," http://www.psfc.mit.edu/library/ja/97ja017 full.html and http://www.psfc.mit.edu/mdsplus/mds plus.html.

[10] Research Systems, Inc., Boulder, Colorado. 